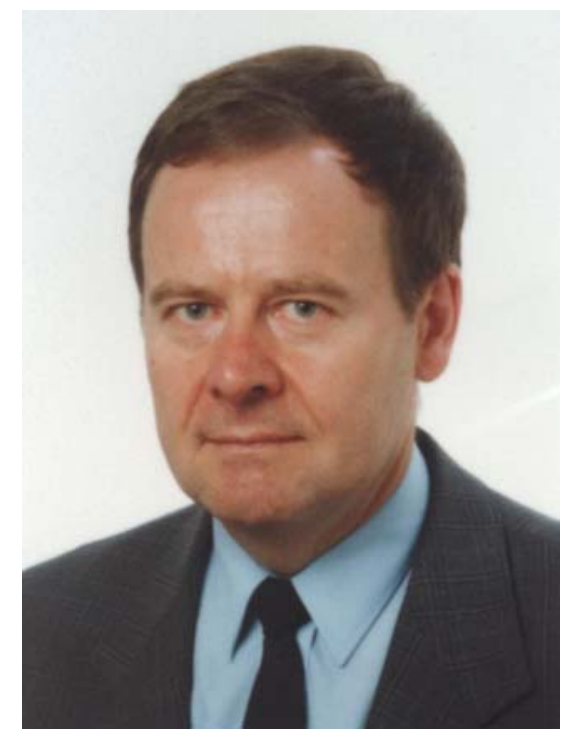

\title{
Prof. Dr. habil. Gerhard Seeland
}

65 Jahre

Gerhard Seeland hat nach einer soliden Aus- und Weiterbildung sein Berufsleben der Tierzuchtwissenschaft gewidmet. Er wurde am 20.06.1941 in einer bäuerlichen Familie in Rutzkau, Krs. Calau, geboren. Es ist uns ein Bedürfnis unserem kollegialen Freund zu seinem 65. Geburtstag sehr herzlich zu gratulieren und ihm alles erdenklich Gute für die Zeit des wohlverdienten Ruhestands zu wünschen. Hiermit verbinden wir unseren tief empfundenen Dank für die effektive, stets fördernde Wirkung, die von ihm für sein Fachgebiet, von den genetischen Grundlagen bis zur Nutzanwendung, ausging.

Seine Ausbildung begann mit dem Besuch der Grundschule in der Heimatgemeinde und anschließender landwirtschaftlicher Lehre, der eine mehrjährige Tätigkeit als Facharbeiter folgte. Nach dieser fundierten Praxisausbildung nahm er das Studium an der Landwirtschaftlichen Fachschule in Cottbus und Schlieben auf, das er 1963 als „Staatlich geprüfter Landwirt“ abschloss, um sich danach der Tätigkeit in der Tierzuchtwissenschaft zu widmen. So begann er als technischer Assistent am Institut für Tierzüchtung und Haustiergenetik der Humboldt-Universität zu Berlin (HUB), der er bis zu seinem Ausscheiden 2005 die Treue hielt.

Das Studium der Landwirtschaftswissenschaften absolvierte Gerhard Seeland als Fernstudent ebenfalls an der HUB und parallel dazu ein Direktstudium für „Mathematische Methoden in der Landwirtschaft“ an der Martin-Luther-Universität Halle. Seine soliden methodischen Kenntnisse kamen vielen Doktoranden von der Versuchsanlage bis zur Ergebnisanalyse zugute. Nach beiden Diplomen wurde er 1969 wissenschaftlicher Assistent am gleichen Institut der inzwischen gebildeten Sektion Tierproduktion und Veterinärmedizin, an der er 1974 mit dem Thema „Zuchtmethodik in den Genreserveherden der Milchrindzüchtung der DDR" mit summa cum laude promovierte.

Bereits 1970 wurden ihm die Vorlesungen und Konsultationen im Fach „Allgemeine Tierzucht“ für Fernstudenten der Tierproduktion übertragen. Später hat er die Vorlesung „Populationsgenetik“ in der postgradualen Ausbildung zum 
„Staatlich anerkannten Tierzuchtleiter“ übernommen. Die qualitative Auswahl der jeweils 30 - 40 Teilnehmer pro Lehrgang und deren mindestens vierjährige, erfolgreiche tierzüchterische Tätigkeit nach dem Studium, bewirkten auch für die Lehrenden erhöhte Anforderungen, die Gerhard Seeland souverän erfüllte.

Er habilitierte 1985 mit einer Arbeit „Zur Schilddrüsenaktivität als Frühselektionskriterium für Merkmale des Wachstums und der Milchleistung beim Rind“. Die Themen der Dissertation und Habilitation vermitteln einen Eindruck von der Breite und Tiefe seiner Forschung sowie seiner daraus resultierenden wissenschaftlichen Kompetenz. Diese spiegelt sich in über 100 Publikationen wider, die vorwiegend in renommierten wissenschaftlichen Zeitschriften des In- und Auslandes erschienen. Hervorzuheben sind die vom VEB Deutscher Landwirtschaftsverlag herausgegebenen Hochschullehrbücher der Tierproduktion „Genetische und physiologische Grundlagen“ und „Züchterische und ökologische Grundlagen“. Beide Bände erschienen 1986 als 2. Lizenzauflage im Verlag Harri Deutsch Thun, Frankfurt/M. und in der Schweiz, was zu diesem Zeitpunkt eher selten war. An beiden Hochschullehrbüchern war Gerhard Seeland überdurchschnittlich stark beteiligt.

Infolge der Trennung der Sektion Tierproduktion und Veterinärmedizin in beide Grunddisziplinen und Wiedereinrichtung der alten Fakultäten (Landw.-Gärtn. und Vet.-med.) übernahm Gerhard Seeland die Vorlesungen „Allgemeine Genetik“ und „Rinderzucht“ an der Veterinärmedizinischen Fakultät der Freien Universität Berlin.

Nach seiner Berufung zum Hochschuldozenten folgte 1993 die zum ordentlichen Professor für „Kleintierzucht“ und 1997 übernahm er die Leitung des Fachgebietes „Züchtungsmethodik und Züchtungsplanung“ an der Landwirtschaftlich-Gärtnerischen Fakultät der Humboldt-Universität zu Berlin.

Seine wissenschaftliche Persönlichkeit bewirkte zahlreiche ehrenamtliche Bindungen, so u.a. als Mitglied im „Ausschuss für genetische Vielfalt“ der Deutschen Gesellschaft für Züchtungskunde, als Mitglied des Vorstandes der deutschen Gruppe der World Poultry Science Association und insbesondere seine Tätigkeit als Redaktionsmitglied der Fachzeitschrift „Archiv für Tierzucht“. Den aktiven Dienst beendete Prof. Seeland in Verbindung mit seinem 65. Geburtstag. Beide Lebensdaten bieten sich an, ihm unsere besondere Wertschätzung für seine zuverlässigen und effektiven Dienste in Lehre, Forschung und Weiterbildung auszusprechen. Dies gleichermaßen im Namen der Studierenden vieler Jahrgänge und der großen Zahl seiner Diplomanden, Doktoranden, Mitarbeiter und Kollegen. Unseren Dank verbinden wir mit den besten Wünschen für schöne Jahre der nahen und fernen Zukunft. 